Revista de Dialectología y Tradiciones Populares, vol. LXXI, n. ${ }^{\circ}$ 1, pp. 49-57, enero-junio 2016, ISSN: 0034-7981, eISSN: 1988-8457, doi: 10.3989/rdtp.2016.01.001.04

\title{
La colaboración como condición: la etnografía participativa como oportunidad para la acción ${ }^{1}$
}

\author{
Collaboration as Condition: Participatory \\ Ethnography as an Opportunity for Action
}

\author{
Luis Berraquero-Díaz \\ Universidad Pablo de Olavide (Sevilla) \\ lberdia@upo.es \\ Francisco Maya-Rodríguez \\ Universidad Pablo de Olavide (Sevilla) \\ curromaya@gmail.com \\ Francisco Javier Escalera Reyes \\ Universidad Pablo de Olavide (Sevilla) \\ fjescrey@upo.es
}

\footnotetext{
${ }^{1}$ Nos gustaría agradecer a los colectivos y las personas que de una forma u otra participaron en los talleres "Sacar los del tiesto", celebrados dentro de las III Jornadas Capital y Territorio, promovidas en 2012 por el Aula de Arte y Pensamiento de la Universidad Internacional de Andalucía. Agradecemos que haya respuestas y propuestas, que día a día, en lo cotidiano, pongan en crisis a la propia crisis, con rabia y creatividad. Particularmente nos gustaría dar las gracias al colectivo Moneda Social Puma por dejarnos colaborar con ellos en ese ejercicio de cuestionamiento colectivo; también a toda la fauna variada que pulula por la Casa Pumarejo y, en especial a Salvador García Guerrero, por creer con firmeza en sus convicciones y vivir conforme a ellas. «Sacar los pies del tiesto" no habría sido posible si no existieran instituciones con cintura; instituciones anómalas que dejan hacer; que dan libertad y fondos a los protagonistas para que se generen procesos colaborativos y recursivos. En este sentido, es necesario agradecer a UNIA arteypensamiento y a BNV Producciones, por su buen hacer y experiencia para crear el ambiente necesario para que emerjan este tipo de procesos. Nuestras últimas gracias van hacia Tomás Sánchez Criado y Adolfo Estalella por organizar el panel "Colaboraciones Experimentales" dentro del I congreso internacional de AIBR y por la labor de coordinación que han realizado para publicar esta serie de artículos.
} 


\section{RESUMEN}

Partiendo de varias experiencias de investigaciones colaborativas llevadas a cabo en Sevilla entre activistas e investigadores, este artículo presenta contextos activistas en los que la colaboración es planteada como una condición sine qua non para llevar a cabo la investigación, analiza las nociones de etnografías colaborativas y los contextos en los que surgen, y reflexiona sobre las coincidencias y diferencias con las propuestas de la investigación social y acción participativa. Por otro lado, propone la etnografía acción participativa como una aproximación que permite: aprovechar la capacidad analítica del método etnográfico para establecer un diálogo mutuo, crítico y horizontal con aquellos que tradicionalmente han sido vistos como "objetos de investigación", favorecer la co-generación de conocimientos situados, incorporar el carácter procesual y la voluntad por la acción transformadora en las investigaciones colaborativas y superar la deriva tecnocrática que reduce la investigación acción participativa a la utilización de técnicas, tecnologías o procedimientos considerados participativos.

Palabras clave: Etnografía acción participativa; Etnografía colaborativa; Investigación acción participativa; Activismo.

\section{SUMMARY}

Drawing on previous experiences of collaborative research carried out in Seville (Spain) by social activists and scholars, this paper presents contexts where collaboration emerges as a sine qua non condition for any research activity. In doing so, we analyse several notions of 'collaborative research' and the contexts for which they were designed and implemented, looking for similarities and differences with participatory action research methods. We propose the participatory action ethnography approach in order to make use of the analytic potential of ethnographic methods, creating a context for mutual, critic and horizontal dialogue with those who have traditionally been regarded as "research objects". This, at the same time, stimulates the co-development of situated knowledges, and incorporates the process-based approach and transforming action in collaborative research, overcoming the 'technocratic drift' which reduces participatory action research to the use of techniques, devices or procedures considered participatory.

Key words: Participatory Action Ethnography; Collaborative Ethnography; Participatory Action Research; Activism.

\section{INTRODUCCIÓN}

Las ideas que presentamos en este artículo surgen a tenor de varias investigaciones realizadas en Sevilla y que han tenido como denominador común la colaboración entre movimientos activistas y el Grupo de Investigación Social y Acción Participativa (GISAP) a lo largo de los últimos 20 años. Se suman a estas colaboraciones, las reflexiones llevadas a raíz de la celebración de las III jornadas sobre capital y territorio, promovidas por el Aula de Arte y Pensamiento de la Universidad Internacional de Andalucía, y celebradas en 2012. Dicho encuentro se centró en el surgimiento o reavivación de iniciativas sociales que plantearan alternativas frente a las políticas de austeridad que comenzaron a implementarse en 2010. Como parte de las mismas, la Red de Moneda Social Puma y la Asociación casa Pumarejo, junto con varios miembros (GISAP) diseñamos un proyecto de investigación colaborativa que se concretó 
en la realización de dos talleres denominados Sacar los Pies del Tiesto y que giraron en torno a dos ejes reflexivos: el sustento y los cuidados. 29 colectivos activistas participaron en estos talleres en los que, por un lado, se analizaron las diferentes significaciones y narrativas que cada uno de los colectivos atribuían a los ejes de reflexión elegidos y, por otro, se evaluaron las prácticas desarrolladas por cada colectivo en referencia a dichos ejes en relación a su capacidad para mitigar la situación de crisis. Redes de trueque, bancos del tiempo, monedas sociales, asambleas barriales del 15M, huertas urbanas - lúdicas y productivas-, cooperativas de economía social, asociaciones culturales, de educación ambiental o de derecho al parto, iniciativas de pedagogía alternativa, colectivos feministas, asociaciones de derechos sociales, fueron algunas de las iniciativas que participaron ${ }^{2}$.

\section{LA ETNOGRAFÍA PARTICIPATIVA COMO NECESIDAD. COLABORACIONES ENTRE EL ACTIVISMO Y LA INVESTIGACIÓN}

Si bien Holmes y Marcus (2008a) ya señalaron que la investigación con y sobre colectivos activistas podría ser un contexto de colaboración etnográfica, consideramos necesario resaltar que, en el caso de ciertos activismos, como los que vamos a comentar a continuación, dicha colaboración entre academia y activistas se convirtió en una condición sine qua non para la realización del proceso de investigación.

La crisis multidimensional ha servido de acción catalizadora hacia un cambio de ciclo en los movimientos sociales tanto en el contexto español como internacional (Azkarraga et al. 2012; García Espín 2012; Haro Barba y Sampedro Blanco 2011; Morán y Limón 2012). En este sentido, se han reavivado, o bien han surgido respuestas de acción colectiva que pivotan entre las protestas creativas, mayoritariamente no violentas en el caso de España (Oliver Olmo y Urda Lozano, 2015) y la generación de estrategias y propuestas cotidianas (Calle 2013; Chatterton y Pickerill 2010; García Espín 2012) que pretenden "superar la crisis" intentando romper la dinámica de "protestarepresión". Entre estas últimas, encontramos iniciativas, como la asociación casa Pumarejo o la red de moneda social Puma — ambos colectivos situados en el norte del casco histórico de Sevilla- o bien algunos de los colectivos que han emergido fruto de ese cambio de ciclo activista, que cuestionan parcial o totalmente, paradigmas del sistema capitalista neoliberal, a través de prácticas cotidianas.

Si bien es cierto que bajo ese paraguas existe una amplia diversidad de iniciativas, encontramos ciertos rasgos característicos que comentamos a continuación. Estos colectivos podrían ser considerados comunidades de prácticas (Lave 1991) que comparten el hecho de entender determinadas parcelas de la vida cotidiana como un escenario fundamental de praxis política. A través de la organización colectiva de los hábitos cotidianos, a los que estos colectivos cargan de significación teórico-política, se ensayan iniciativas productivas, reproductivas, económicas, sociales, políticas, habitacionales

\footnotetext{
${ }^{2}$ Para ampliar información sobre las jornadas que aquí se mencionan, puede visitarse la siguiente web http://ayp.unia.es/index.php?option=com_content\&task=view\&id=742 en la que puede descargarse el informe elaborado en conjunto entre las diversas personas que formaron parte de ese equipo de investigación colaborativa.
} 
con un enfoque pragmático. Estas iniciativas, al menos en el plano discursivo, dicen estar basadas en paradigmas diferentes a los del sistema capitalista neoliberal, aunque no por ello exentas de contradicciones. Dichas contradicciones, unidas a una tendencia al auto-análisis, seguimiento y evaluación de sus propias prácticas, contribuyen a desarrollar un corpus teórico-práctico. Esto genera por un lado narrativas propias, matizadas y situadas con respecto al conocimiento académico y, por otro lado, un empoderamiento sobre su proceso de conocimiento.

Esa consideración de experticia auto-percibida junto con el proceso de empoderamiento colectivo que se deriva de esa toma de conciencia, contribuye a que estos colectivos pongan en tela de juicio la centralidad de la academia en la producción de conocimiento experto, frente a otras comunidades de conocimiento. Es en este sentido, que decimos la colaboración es una condición para la puesta en marcha de procesos de investigación y no tanto una concesión que emana desde los sujetos que investigan.

\section{INVESTIGACIONES PARTICIPATIVAS Y ETNOGRAFÍAS COLABORATIVAS ¿OPORTU- NIDADES PARA LA ACCIÓN TRANSFORMADORA?}

La realización de etnografías colaborativas (EC) surge a tenor de las potencialidades encontradas entre comunidades epistémicas donde hay sujetos que cuentan ya con mecanismos reflexivos, capaces de analizarse por y para sí mismos; sin necesidad de un etnógrafo que ordene, valore o sitúe estos relatos ante un público concreto (Holmes y Marcus 2008a, 2008b). El enfoque colaborativo y participativo en investigación comparte el interés por diluir o abarcar mejor la complejidad de las clásicas dualidades sujeto/objeto, nosotros/otros o emic/etic (Banerjea 2015), así como por facilitar el establecimiento de un diálogo mutuo, crítico y horizontal con aquellos que tradicionalmente han sido vistos como objetos de investigación" (Friedman 2013). En ciencias sociales, lo participativo, frente a lo participante, se ha venido diferenciando por el cambio radical en la relación sujeto-objeto de la investigación. Sin embargo, la legitimidad y el éxito de la incorporación de lo participativo a procesos de investigación ha derivado, en ocasiones, hacia una utilización ambigua por parte de instituciones públicas y colectivos sociales. Así, la participación ha sido presentada como mecanismo de transparencia o democratización aparentes sin que por ello se garantice la apertura real y efectiva de procesos participativos con capacidad de influencia en la toma de decisiones. (De la Riva y Moreno 2015). Deseamos que no ocurra lo mismo con el concepto de lo colaborativo.

Por otra parte, las EC han sido identificadas como una forma de establecer compromisos personales, políticos y profesionales entre investigadores y comunidades dirigidos a la acción social transformadora (Banerjea 2015; Madison 2005). Es precisamente en este punto donde las EC coinciden con la tradición de la investigación-acción participativa (IAP). La IAP es un enfoque epistemológico con un corpus teóricometodológico en el que "los dueños locales de un problema" (Greenwood 2000: 36) colaboran junto con un grupo de personas investigadoras-dinamizadoras para poner en marcha un proceso co-generativo de conocimiento. Este proceso está enfocado a proponer intervenciones a corto plazo, así como estrategias transcontextuales de ac- 
ción a largo plazo que den continuidad al proceso; un proceso cuya meta final es la transformación social. La IAP pretende pasar de la subordinación a la igualdad construyendo un sujeto colectivo que es, a la vez, investigador e investigado, y que, a diferencia de las EC, además utiliza el proceso de investigación para la toma de conciencia y la generación de propuestas de acción para la transformación. No obstante, la IAP ha derivado en ocasiones hacia puro activismo con escasa o nula investigación y reflexión; o bien hacia enfoques paternalistas en los que existe producción de conocimiento para la acción, pero con escasa o nula participación del colectivo cuya realidad se pretende transformar (Greenwood 2000). La reciente y muy necesaria compilación sobre metodologías participativas publicada por la Red CIMAS da cuenta de hasta qué punto este corpus de praxis metodológicas han evolucionado, incorporando aportaciones que en las últimas décadas han realizado los feminismos, el pensamiento decolonial o las ciencias de la sostenibilidad.

Así pues, encontramos muchas coincidencias entre los planteamientos de la IAP y las EC. Son múltiples los contextos susceptibles de generar discursos y prácticas que podríamos llamar paraetnográficos (Holmes \& Marcus 2008b), como ha mostrado Lassiter (2005). No obstante, ha de tenerse en cuenta que ambas metodologías provienen de campos y tradiciones muy distintos, con horizontes epistémicos poco comparables. Si bien el marco colaborativo se propone con fines técnicos, éticos, académicos y epistémicos, la IAP persigue metas transformadoras. Para ello, además del protagonismo de los actores sociales en el diseño, desarrollo del proceso de investigación y elaboración de sus resultados, es imprescindible que exista voluntad de acción por parte del sujeto colectivo.

Sin menoscabo de lo anterior, los objetivos del proyecto, los requerimientos temporales o aquellos que vengan dados por el tipo de financiación también ejercerán influencia (Banerjea 2015; Cheney 2011; Friedman 2013; Holmes y Marcus 2008a). Desde nuestro punto de vista, la suma de estos factores hace de la colaboración una estrategia metodológica que tendrá que ser valorada por el equipo investigador, en función de las características del campo donde se dispone a integrarse y las condiciones de posibilidad de su investigación.

\section{ETNOGRAFÍA ACCIÓN PARTICIPATIVA COMO PROPUESTA}

Si las etnografías colaborativas ya representan un desarrollo minoritario, son excepcionales los ejemplos de procesos de investigación etnográfica en los que se llega a producir este salto paradigmático, adquiriendo el carácter de Etnografía Acción Participativa (EAP). Entre los pocos ejemplos de intento de la integración de la etnografía colaborativa en un proceso de IAP está el trabajo desarrollado por Campbell y Lassiter (2010) en el proyecto The Other side Of Middletown en la localidad de Mucie (Indiana), y el de Martha Isabel Bernal con la población nasa en la ciudad de Bogotá (2012). Existen además algunas experiencias donde se han llevado a cabo estudios etnográficos de carácter colaborativo conectados de alguna manera con procesos de IAP, ya sea como etapa previa o posterior al desarrollo de los mismos. Encontramos ejemplos relacionados con procesos de patrimonialización (Hollowell y Nicholas 2009; Pyburn 2009), en producciones artísticas (Friedman 2013), o en los campos de la edu- 
cación (Villenas 2007; Van Sluys 2010; Fuentes 2013; García, A. et al. 2015) y de la salud (Goepp et al. 2008; Nugus et al. 2012; Varcoe et al. 2013; Gorli et al. 2015; Oye et al. 2015).

No obstante, la idea de la EAP no es tan reciente. Mariño (2006) la plantea como una alternativa necesaria para superar la falta de incidencia de la etnografía convencional en la transformación de las condiciones de vida de los "dueños del problema", así como para superar la deriva "activista" de la IAP, reducida muchas veces a mera acción participativa. Junto a esto, nosotros añadimos otra deriva, la "tecnocrática", que reduce la IAP a la utilización de técnicas, tecnologías o procedimientos considerados participativos, a menudo empleados para sustentar decisiones políticas que poco o nada reflejan los intereses, necesidades y objetivos de los colectivos ni su transformación.

Por otro lado, las limitaciones que plantea la EAP son muchas, algunas compartidas con las que pueda tener cualquier proceso de IAP en sentido estricto. De todas ellas, quizás la limitación troncal es que la EAP resulta completamente dependiente de las dinámicas del contexto donde se despliega. Si bien un contexto como el que hemos descrito al inicio puede ser favorable o incluso determinante para establecer un proceso de EAP, debemos ser conscientes de que este tipo de situaciones no son la norma. No todos los colectivos tienen el grado de experticia y disposición hacia el auto-análisis como el de los activismos que aquí hemos presentado. No queremos decir con esto que la EAP se deba circunscribir exclusivamente a comunidades epistémicas con cierta o, como en nuestro caso, mucha conexión con la academia. Es más, creemos que son muchos los contextos que funcionan al margen de lo normativo donde afloran los procesos paraetnográficos. Por otro lado, la EAP no tiene cabida en situaciones donde no es posible proporcionar un entorno de libre expresión para todos los participantes. Si bien, este tipo de escollo es fácilmente identificable en situaciones donde las relaciones de dominación son flagrantes, es en situaciones de "aparente libertad" donde las dinámicas internas del contexto plantean mayores retos. Tal y como Friedman describe durante el proceso de un rodaje etnográfico colaborativo, "los miedos y ambivalencias de las familias participantes a expresar lo que sentían, hicieron difícil distinguir qué aspectos no pueden decirse, qué aspectos se rehúsan a decir, o simplemente ni siquiera mencionan, en una etnografía colaborativa" (Friedman 2013: 391) Un proceso de EAP implica analizar los conflictos evidentes, pero sobre todo los latentes, los que no se mencionan. La negociación en cada una de las fases del proceso emerge así como una herramienta en la que se evidencian los choques y potencialmente pueden complejizarse los significados (Madison 2005).

\section{CONCLUSIONES}

La EAP posee evidentes potencialidades para llevar a la práctica los principios que sustentan a la IAP o para ser implementada en investigaciones colaborativas con vocación transformadora. Sin duda se trata de una metodología vinculada de modo indisociable a contextos específicos en los que poder desarrollar una observación directa, vínculo que implica mapear las relaciones de los sujetos colaboradores que participan del proceso de investigación. Esto facilita la integración del análisis de las relaciones de poder existentes entre los diferentes sujetos de investigación y las que, 
a su vez, éstos establecen con la comunidad académica, los colectivos a los que pertenecen o los organismos que financian el proceso. En definitiva, la metodología etnográfica permite trazar el campo de relaciones de poder que afectan a la definición, la conducta, los resultados y la presentación de informes del proyecto etnográfico (Horner 2002: 14).

Fruto de la convivencia prolongada dentro del propio fenómeno investigado, la etnografía genera un conocimiento empático, profundo y con una fuerte validez interna. La etnografía colaborativa, sustentada además en algunos de los fundamentos epistemológicos que caracterizan la llamada "mirada antropológica" (holismo, relativismo cultural, empatía y extrañamiento, por ejemplo), consigue de este modo el suficiente grado de profundización y fineza en el proceso de producción de conocimiento y de análisis de la realidad por parte de los integrantes del colectivo. Por otra parte, si bien este conocimiento resulta clave a la hora de articular decisiones y acciones a corto y largo plazo, su elaboración requiere mucho tiempo, y a menudo procesos de formación y aprendizaje de diversa índole, así como esfuerzos constantes de coordinación.

En este sentido, la EAP implica un viraje en las metas epistémicas de la etnografía. La vertiente clásica del método entiende la fase explicativa de la investigación como la interpretación de hechos empíricos constatados por un etnógrafo que, en cierto modo, actúa como pontífice entre academia y sociedad. Lo que permite la EAP es la propagación de esa interpretación-explicación a lo largo de una red completa de actores participantes. En este sentido, los etnógrafos pasan de ser traductores a convertirse en vértices de una red que integra academia y sociedades.

\section{BIBLIOGRAFÍA CITADA}

Azkarraga, Joseba, Tod Sloan, Patricio Belloy y Aitzol Loyola. 2012. "Eco-localismos y resiliencia comunitaria frente a la crisis civilizatoria. Las Iniciativas de transición". Polis, Revista de la Universidad Bolivariana 11(3): 315-40.

Banerjea, Niharika. 2015. "Critical Urban Collaborative Ethnographies: Articulating Community with Sappho for Equality in Kolkata, India”. Gender, Place and Culture 22(8): 1058-1072. http://doi.org/ 10.1080/0966369X.2014.939145

Bernal, Martha I. 2012. "Territorialidad nasa en Bogotá: apropiación, percepción y sentido de lugar". Cuadernos de Geografía. Revista Colombiana de Geografía 21(1): 83-98.

Calle, Ángel. 2013. La transición inaplazable. Salir de la crisis desde los nuevos sujetos politicos. Barcelona: Icaria.

Campbell, Elizabeth y Luke E. Lassiter. 2010. "From Collaborative Ethnography to Collaborative Pedagogy: Reflections on the Other Side of Middletown Project and Community-University Research Partnerships". Anthropology \& Education Quarterly 41(4): 370-385. http://doi.org/ 10.1111/j.1548-1492.2010.01098.x

Red CIMAS (eds.) 2015. Metodologias participativas: sociopraxis para la creatividad social. Madrid: Dextra Editorial.

Chatterton, Paul y Jenny Pickerill. 2010. "Everyday Activism and Transitions Towards Post-Capitalist Worlds". Transactions of the Institute of British Geographers, 35(4): 475-490. http://doi.org/ 10.1111/j.1475-5661.2010.00396.x

Cheney, Kristen E. 2011. "Children as Ethnographers: Reflections on the Importance of Participatory Research in Assessing Orphans' Needs". Childhood 18(2): 166-179. http://doi.org/10.1177/ 0907568210390054

De la Riva, Fernando y Antonio Moreno. 2015. “Por qué lo llaman participación cuando quieren decir comparsa?". El Topo Tabernario, 13: 10-11. 
Friedman, P. Kerim. 2013. "Collaboration against Ethnography: How Colonial History Shaped the Making of an Ethnographic Film". Critique of Anthropology 33(4): 390-411. http://doi.org/10.1177/ $0308275 \times 13499385$

Fuentes, Emma. 2013. "Political Mothering: Latina and African American Mothers in the Struggle for Educational Justicen. Anthropology \& Education Quarterly 44(3): 304-31. http://doi.org/10.1111/ aeq.12027

García Espín, Patricia. 2012. "El 15M/ : de vuelta al barrio como espacio de lo político". Revista Internacional de Pensamiento Político 7: 291-310.

García, Antero, Nicole Mirra, Ernest Morrell, Antonio Martínez y D’Artagnan Scorza. 2015. "The Council of Youth Research: Critical Literacy and Civic Agency in the Digital Age». Reading \& Writing Quarterly 31(2): 151-167. http://doi.org/10.1080/10573569.2014.962203

Goepp, Julius G., Tiesha Johnson y Charles Maddow. 2008. "Challenges and Promises for Nurse Education Curriculum Development in Kosovo: Results of an 'Accidental Ethnography', Nurse Education Today 28(4): 419-426. http://dx.doi.org/10.1016/j.nedt.2007.07.002

Gorli, Mara, Davide Nicolini y Giuseppe Scaratti. 2015. "Reflexivity in Practice: Tools and Conditions for Developing Organizational Authorship". Human Relations 68(8): 1347-1375.

Greenwood, David. 2000. "De la observación a la investigación-acción participativa: una visión crítica de las prácticas antropológicas". Revista de Antropología Social 9: 27-49.

Haro Barba, Carmen y Víctor Sampedro Blanco. 2011. "Activismo político en red: del movimiento por la vivienda digna al 15M. Teknokulturaw. Revista de Cultura Digital y Movimientos Sociales 8(2): 167-185. http://doi.org/ISSN 15492230

Hollowell, Julie y Nicholas George. 2009. "Using Ethnographic Methods to Articulate CommunityBased Conceptions of Cultural Heritage Management. Public Archaeology: Archaeological Ethnographies 8(2-3): 141-160. http://doi.org/10.1179/175355309X457196

Holmes, Douglas R. y George E. Marcus. 2008a. "Collaboration Today and the Re-Imagination of the Classic Scene of Fieldwork Encounter". Collaborative Anthropologies, 1(1): 81-101. http:// doi.org/10.1353/cla.0.0003

Holmes, Douglas R. y George E. Marcus. 2008b. "Para-Ethnography", en Lisa Given (ed.), The SAGE Encyclopedia of Qualitative Research Methods: 26-27. Londres: Sage.

Horner, Bruce. 2002. "Critical ethnography, ethics, and work: Rearticulating labor". Journal of Advanced Composition 22(3): 561-584.

Lave, Jean. 1991. "Situating learning in communities of practice", en Lauren B. Resnick, John M. Levine y Stephanie D. Teasley (eds.), Perspectives on Socially Shared Cognition: 63-82. Washington: American Psichological Association.

Madison, D. Soyini, 2005. "Introduction to critical ethnography: Theory and Method", en Madison D. Soyini, Critical Ethnography: Method, Ethics, and Performance: 1-16. Londres: Sage. http:// dx.doi.org/10.4135/9781452233826.n1

Mariño, Germán. 2006. "El diálogo en educación: Recapitulaciones sobre la construcción de una propuesta pedagógica", en Juan C. Echeverri y Julio Bernal (eds.), Territorios pedagógicos: espacios, saberes y sujetos: 89-116. Bogotá: Universidad Pedagógica Nacional.

Morán, Carmen y Raúl Limón. 2012. Activismo efervescente. El Pais, 8 Agosto: 10-13. Disponible en: <http://sociedad.elpais.com/sociedad/2012/08/08/actualidad/1344453806_680343.html>. Fecha de acceso: 12 feb. 2016.

Nugus, Peter, David Greenfield, Joanne Travaglia y Jeffrey Braithwaite. 2012. "The Politics of Action Research: 'If You Don't Like the Way Things are Going, Get Off the Bus'. Social Science E Medicine 75(11): 1946-1953. http://dx.doi.org/10.1016/j.socscimed.2012.06.024

Oliver, Pedro y Jesús C. Urda. 2015. Protesta democrática y democracia antiprotesta. Navarra: Pamiela. Øye, Christine, Tone E. Mekkin, Randi Skaar, Hellen Dahl, Oddvar Forland y Frode F. Jacobsen. 2015. "Evidence Molded by Contact with Staff Culture and Patient Milieu: An Analysis of the Social Process of Knowledge Utilization in Nursing Homes". Vocations and Learning 8(3): 319-334. http:/ /doi.org/10.1007/s12186-015-9135-2

Pyburn, K. Anne. 2009. "Practising Archaeology-As if it Really Matters". Public Archaeology: Archaeological Ethnographies 8(2-3): 161-175. http://doi.org/10.1179/175355309X457204 
Van Sluys, Katie. 2010. «Trying On and Trying Out: Participatory Action Research as a Tool for Literacy and Identity Work in Middle Grades Classrooms". American Journal of Community Psychology 46:139-151. http://doi.org/10.1007/s10464-010-9319-8

Varcoe, Colleen, Helen Brown, Betty Calam, Thelma Harvey y Miranda Tallio. 2013. "Help Bring Back the Celebration of Life: A Community-Based Participatory Study of Rural Aboriginal Women's Maternity Experiences and Outcomesn. BMC Pregnancy and Childbirth 13(26). http://doi.org/ $10.1186 / 1471-2393-13-26$

Villenas, Sofia A. 2007. "Diaspora and the Anthropology of Latino Education: Challenges, Affinities, and Intersections". Anthropology \& Education Quarterly 38(4): 419-425. http://doi.org/10.1525/ aeq.2007.38.4.419 